Corrigendum

\title{
Corrigendum to "Local and Systemic Cardiovascular Effects from Monochromatic Infrared Therapy in Patients with Knee Osteoarthritis: A Double-Blind, Randomized, Placebo-Controlled Study"
}

\author{
Ru-Lan Hsieh, ${ }^{1,2,3}$ Wei-Cheng Liao, ${ }^{2}$ and Wen-Chung Lee ${ }^{4}$ \\ ${ }^{1}$ Department of Physical Medicine and Rehabilitation, Shin Kong Wu Ho-Su Memorial Hospital, 95 Wen-Chang Road, \\ Shih-Lin District, Taipei 111, Taiwan \\ ${ }^{2}$ Department of Physical Medicine and Rehabilitation, School of Medicine, College of Medicine, Taipei Medical University, \\ 250 Wuxing Street, Taipei 110, Taiwan \\ ${ }^{3}$ School of Nursing and Management in Gerontology, College of Nursing, Taipei Medical University, 250 Wuxing Street, \\ Taipei 110, Taiwan \\ ${ }^{4}$ Institute of Epidemiology and Preventive Medicine, College of Public Health, National Taiwan University, 17 Xuzhou Road, \\ Taipei 100, Taiwan
}

Correspondence should be addressed to Ru-Lan Hsieh; m001052@ms.skh.org.tw

Received 2 January 2017; Accepted 19 January 2017; Published 14 March 2017

Copyright (C) $2017 \mathrm{Ru}$-Lan Hsieh et al. This is an open access article distributed under the Creative Commons Attribution License, which permits unrestricted use, distribution, and reproduction in any medium, provided the original work is properly cited.

In the article titled "Local and Systemic Cardiovascular Effects from Monochromatic Infrared Therapy in Patients with Knee Osteoarthritis: A Double-Blind, Randomized, Placebo-Controlled Study" [1], affiliation number two was incomplete. The corrected affiliation is shown above.

\section{References}

[1] R.-L. Hsieh, W.-C. Liao, and W.-C. Lee, "Local and systemic cardiovascular effects from monochromatic infrared therapy in patients with knee osteoarthritis: A Double-blind, Randomized, Placebo-controlled Study," Evidence-based Complementary and Alternative Medicine, vol. 2012, Article ID 583016, 9 pages, 2012. 


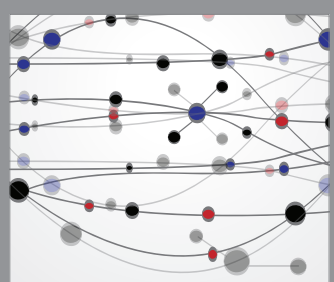

The Scientific World Journal
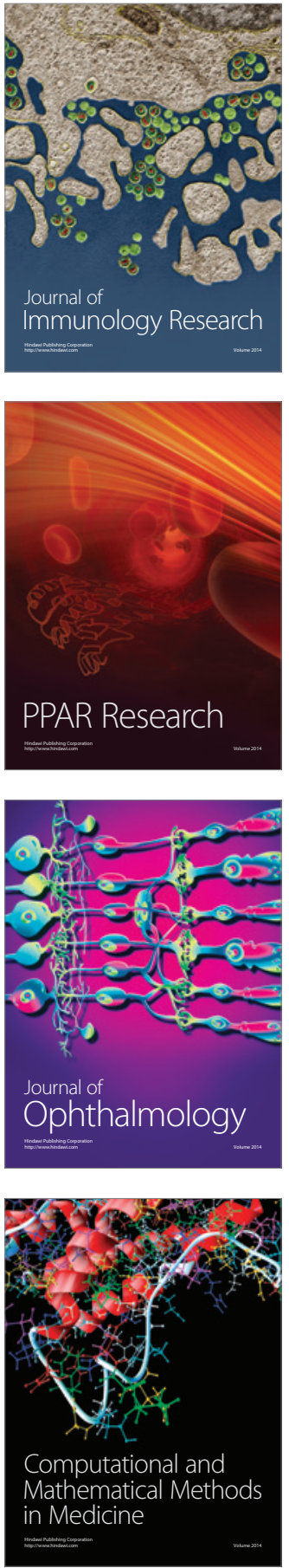

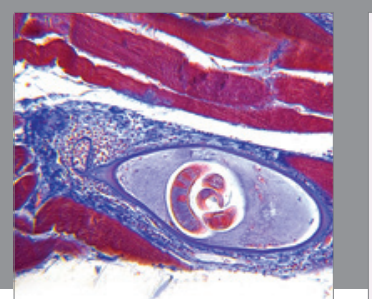

Gastroenterology Research and Practice
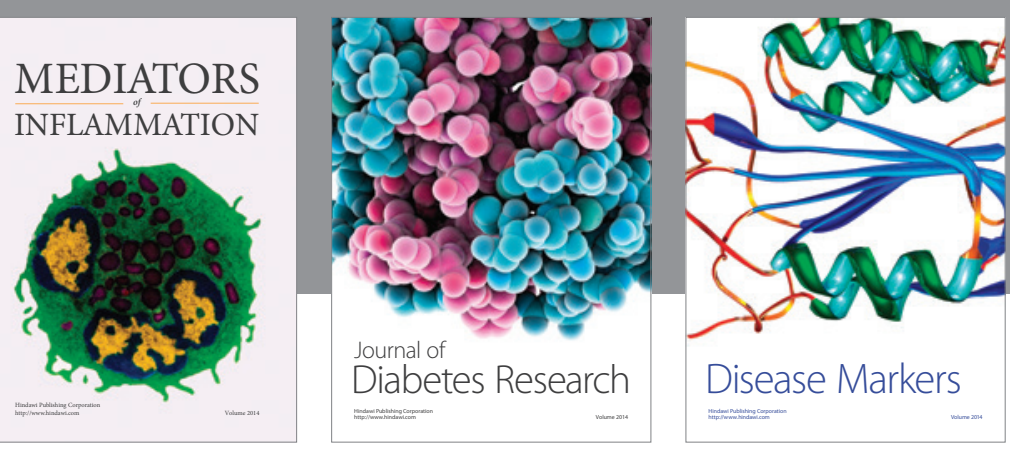

Disease Markers

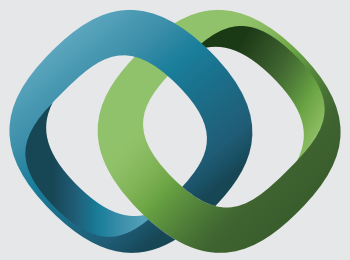

\section{Hindawi}

Submit your manuscripts at

https://www.hindawi.com
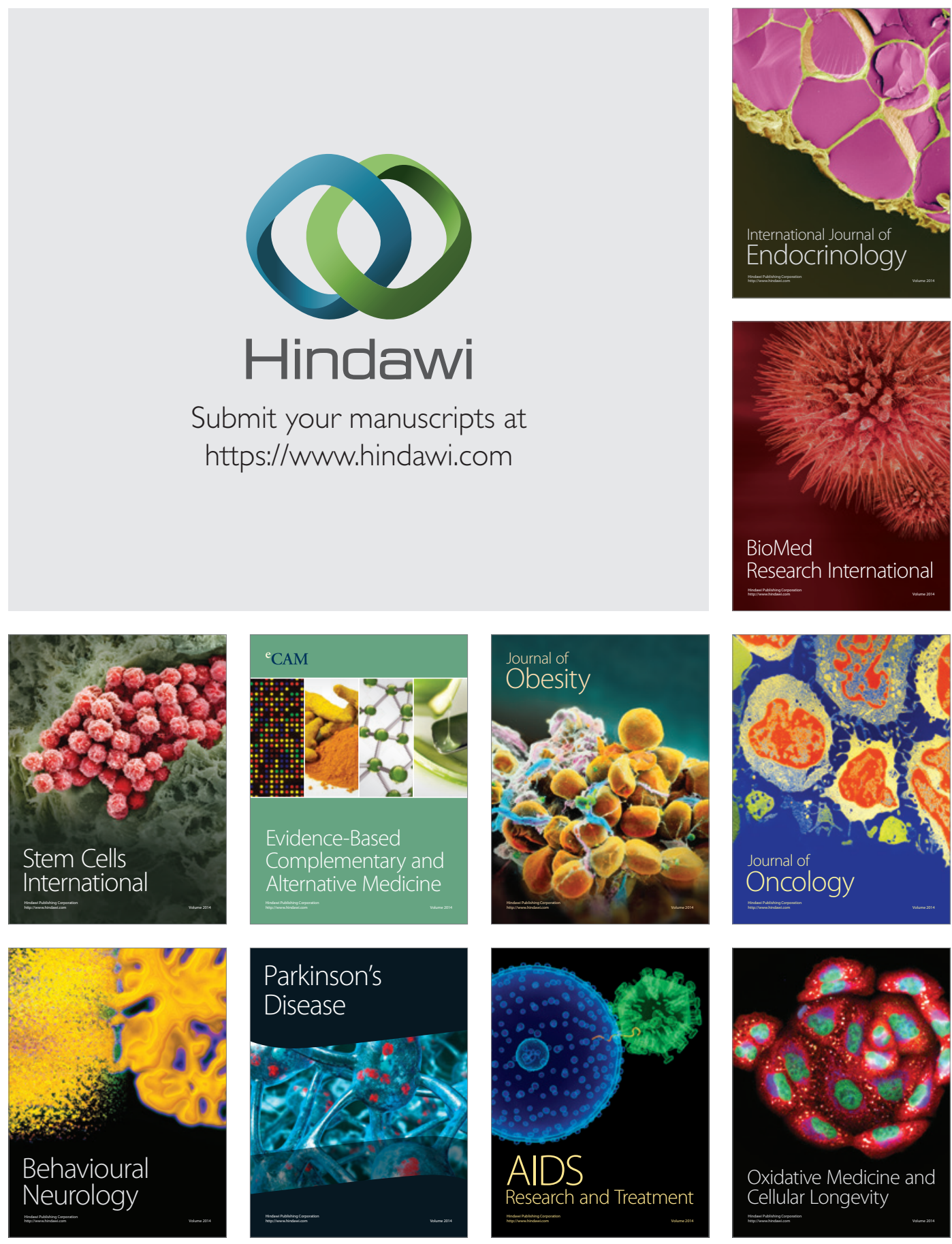